\title{
MULTI-SCALE SPATIAL MODELLING OF ELECTRIC POWER DISTRIBUTION NETWORKS
}

\author{
Yaping Hua ${ }^{1,2, *}$, Guiwen Lan ${ }^{1,2}$, Yongliang $\mathrm{Du}^{1,2}$ \\ ${ }^{1}$ College of Geomatics and Geoinformation, Guilin University of Technology, Guilin, China \\ ${ }^{2}$ Guangxi Key Laboratory of Spatial Information and Geomatics, Guilin University of Technology, Guilin, China
}

KEY WORDS: Electric Power Distribution Network (EPDN), Multi-Scale, Spatial Modelling, Graphic Data, Attribute Data

\begin{abstract}
:
The research proposes a multi-scale spatial data model of electric power distribution networks (EPDNs) to address the problem that the single-scale EPDN data cannot meet the needs of data representation and spatial analysis of multiple levels of detail (LODs). This study comprehensively analyses the equipment used in the EPDN, summarizes the detailed information of EPDN elements and constructs a reasonable EPDN structure system. Based on the analysis of a large number of use cases in the operation and maintenance field of EPDN, this research identifies the elements of the graphic data and attribute data relating to the EPDN. According to the needs of different users and different application modes of EPDN data in multi-scale data representation, the EPDN data models are divided into four LODs, and the simplification principle of constructing different LODs is put forward and the elements contained in each LOD are carefully modelled. This study divides the EPDN elements information into graphic attribute and functional attribute, and then the attribute information of the EPDN data models in different LODs is described in detail. In addition, the EPDN data of Yan Shan campus of Guilin University of Technology is modelled with the proposed method, which has achieved good visualization results.
\end{abstract}

\section{INTRODUCTION}

EPDNs are the networks consisting of power lines and distribution equipment responsible for distributing electric power energy (Cao, 2017). With the vigorous development of smart city and intelligent power grid, single-scale EPDNs data is increasingly unable to meet the needs in visual expression and spatial analysis. It is becoming more and more important to build EPDNs data models suitable for multi-scale expression. EPDNs have a wide distribution range, and the spatial relationship and structure of the constituent elements are relatively complex. At present, there are many ways to conduct $3 \mathrm{~d}$ models of EPDNs, such as surface-based patch structures (Zhu et al., 2007), and voxel-based irregular tetrahedral structures (Wu et al., 2005) and so on. However, it is basically to abstract the spatial entity elements into two categories: the electric power points and electric power lines. At small scales, this expression of EPDNs is understandable, but at large scales, this method of generalization of electric power point elements easily ignores the geometric composition of complex ancillary facilities in reality, resulting in their inaccurate spatial relations with other entity elements and incomplete semantic information.

At present, the researches on multi-scale are mostly focused on LOD classification of models and automatic generation algorithm of models with different detail levels (Wu et al., 2006) (Liu, 2005). Hu et al. (2006) proposed to establish LOD models by dividing geographical entities into point elements line elements, area elements, note elements and grid elements, but she did not describe how to express the attribute information of different categories entities. Zhao (2013) put forward an automatic simplification method of multi-detail levels for complex 3D building models, which took into account the geometry, topology, semantics and other details of complex 3D building models, but it was limited to the complex buildings

\footnotetext{
* Corresponding author e-mail: 2272133181@qq.com
}

and needed to be further studies in EPDNs. Qu et al. (2018) studied the $3 \mathrm{D}$ simulation of electric power transmission equipment based on LOD technology, and constructed the LODs of the model, but it only involved a simplified method of fewer parts. On the basis of the current technical specifications (Ningbo, 2012) and data standards (Suzhou, 2007, Jiangsu, 2017, Shenzhen, 2015) for urban underground pipeline detection, this paper comprehensively and systematically combs the constitution system of EPDNs, summarizes the graphic information and attributive information of constituent elements effectively, considers the importance of graphics and attributes of electric power equipment in different use case scenarios, and divides the EPDNs models into four LOD levels. On this basis, this paper puts forward the simplified principles of different levels of detail for the EPDNs, and constructs different EPDNs models.

\section{ANALYSIS AND RESEARCH ON MODELLING ELEMENTS OF EPDNS}

\subsection{EPDNS MODELLING ELEMENTS}

At present, the underground pipeline detection technical regulations and mapping technical specifications are used for surveying and mapping, the data standards for various types of electric power lines are also used to classify the constituents of EPDNs for the purpose of facilitating marking and annotation, even including some ancillary facilities that belong to the scope of surveying but not actually exist. In this paper, according to the function of the components of EPDNs, the EPDNs elements can be divided into three categories: electric power points (such as electric power cable accessories and ancillary facilities), electric power lines (such as overhead power lines and electric power cable), and building structures. The research objects comprise 3 kinds of electric power cable accessories, 17 kinds of ancillary facilities, 2 kinds of electric power lines and 3 kinds of large-scale building structures of EPDNs, which form the structural system of EPDNs. 
A lot of electric power pipe fittings are connected to the outer sleeve of the cables or electric power pipes, which connected to the cables is called electric power cable accessories, mainly including power cable terminal, power cable intermediate connector and power cable $\mathrm{T}$ connector. Electric power ancillary facilities are an important type of equipment in EPDNs, mainly including electric gate boxes, street lamps, street lamp control boxes, power distributing cabinets, lighting distribution boxes, telegraph poles, transformers, cable branch boxes, billboards, joint boxes, control cabinets, signal lamps, Straight-through cable wells, three-way cable wells, four-way cable wells, cable manhole wells, compensators. They are responsible for a lot of important tasks, such as the distribution of electric energy to different branches of the lines, and the control of line switches. The electric power lines are divided into overhead power lines and electric power cables, and the electric power cables are further divided into service cables, street lamp cables, traffic signal cables, tram cables, advertising cables, green lighting cables, communication cables. Building structures are a kind of place in EPDNs that bear important functions, such as power transformation and distribution, mainly including transformer substations, switch board rooms, and switch cabinets. The transformer substations also include primary equipment such as transformers, high-voltage circuit breakers, isolating switches, bus bars, lightning arresters, capacitors, and reactors.

\subsection{ANALYSIS OF EPDNS STRUCTURE}

In order to better show the relationship between EPDNs and the elements they contain, the class diagram is used to express the relationship. Figure 1 accurately describes the constituent system of EPDNs and the attribute information of modelling elements.

This paper divides the electric power points into power-

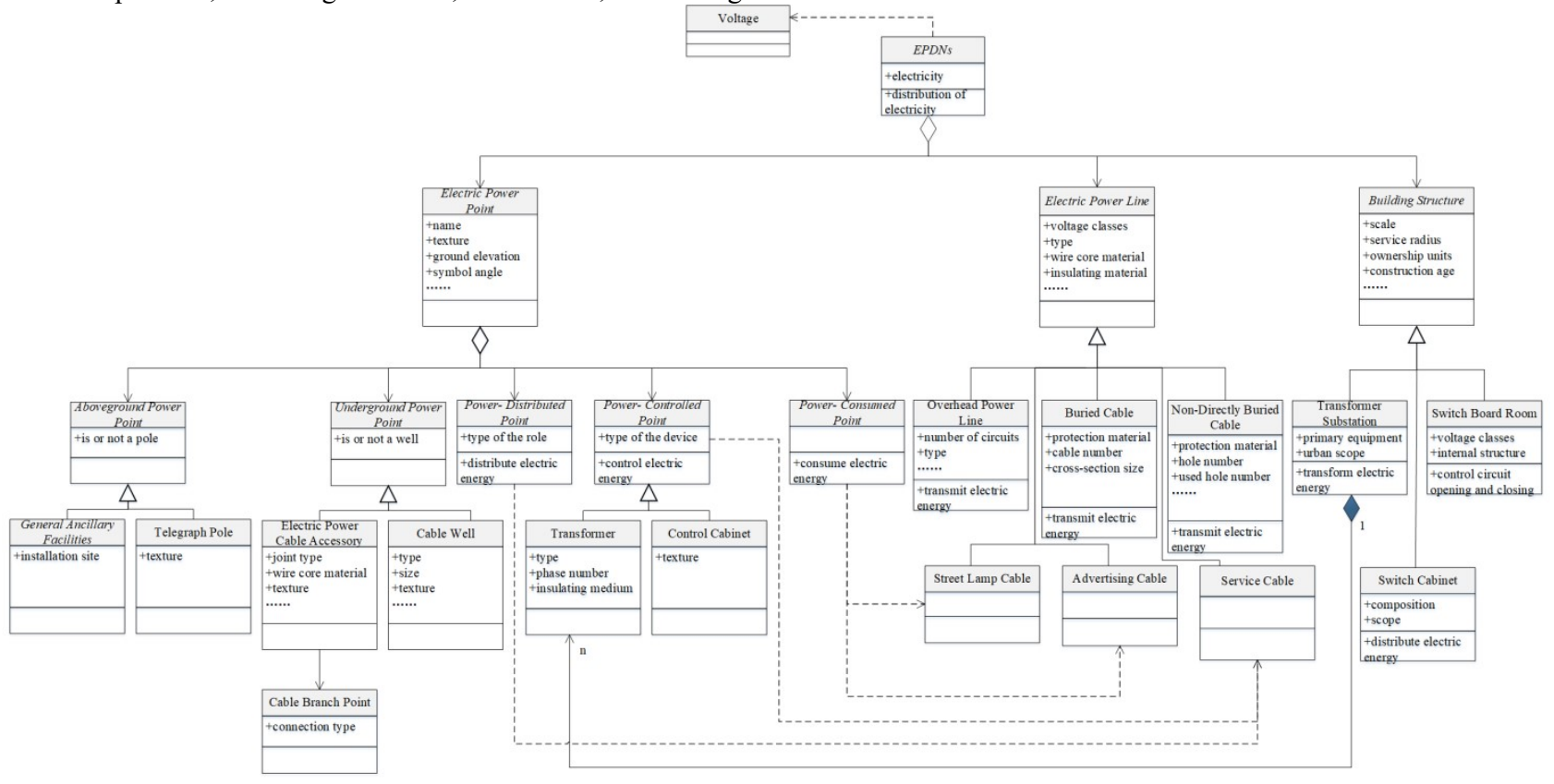

Figure 1. Structure system of EPDNs

distributed points, power-controlled points and power-consumed points according to the purpose. The point elements of EPDNs can be divided into aboveground electric power points and underground electric power points according to the spatial position of the model. According to the buried mode, the electric power line elements are classified into overhead power lines, buried cables and non-directly buried cables, and according to the type of power lines, they can be divided into service cables, street lamp cables, advertising cables(the type of cables included in the campus). Among them, the power- distributed equipment and power-controlled equipment of the electric power points need to be connected with the service cables, and the power-consumed equipment need to be connected with the street lamp cables and advertising cables. The relationship between them is interdependent. Transformer substations, switch board rooms and switch cabinets inherit building structures. The electric power cable accessories exist in each cable branch points, and they are related. Transformers must exist in transformer substations, but not only in transformer substations, and they are a combination relationship.
The spatial entities in geography do not exist in isolation, but have a certain spatial connection relations. The description of the spatial connection relation between the electric power elements is called topological relation, and the topological relation between all target bodies is generally called topological structure. EPDNs also have a certain topological relationship. For example, the cable branch boxes are mainly used to branch the trunk cables and divide them into many small-area cables, which are generally located at the main branch points where the electrical load is big. The electric gate boxes are used to control the household circuits, and the distributing cabinets can control the circuits of a housing estate, so the distributing cabinets can control the electric gate boxes, and the electric gate boxes are generally disposed in the branch circuits of the distribution cabinets. A cable well is a passage for convenient excavation of an overhaul, threading or lengthening cables. It is usually located at a cable turn or cable joint, and the power cable intermediate connectors and power cable $\mathrm{T}$ connectors are generally set in the cable wells. Figure 2 shows the three-way cable well and its internal structure. 

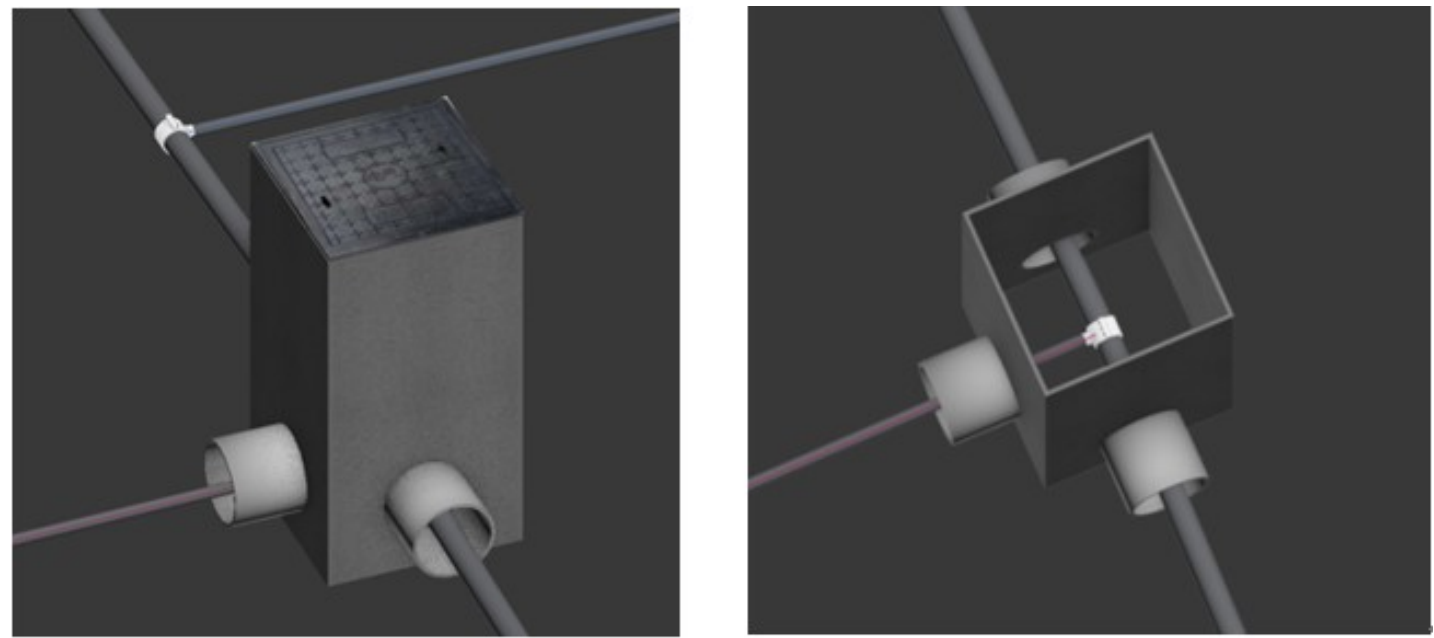

Figure 2. Three-way cable well

\section{MULTI-SCALE SPATIAL MODELLING OF EPDNS}

\subsection{SIMPLIFICATION PRINCIPLE OF DIFFERENT LODS FOR EPDNS}

The essence of multi-scale expression of spatial entity features is that when the size of the visual scene is fixed, the larger the spatial scale, the fewer and finer the displayed features; the smaller the spatial scale, the more and coarser the displayed features. In other words, according to the importance of the elements, the elements of different scales are divided into different display levels, namely the LOD levels. However, too many LOD levels are divided, resulting in multiple sets of model data in different detail levels, which is manifested as data redundancy and low visual efficiency; too few LOD levels are divided, which will leads to obvious mutation phenomenon of model switching between different detail levels. At present, there are no uniform standards for the establishment of multiple LOD models of entity elements in different industries. Buildings with more researches are generally divided into five LOD levels (Zhu et al., 2005, Zhou et al., 2006). The establishment of EPDNs LOD models is related to the graphic accuracy and complexity of different elements in different levels. When constructing EPDNs model, it is necessary to consider the demands of users with different background, on the other hand, due to the EPDNs models in different fields have different application modes, their demand for details expression is also need to consider.

In order to ensure the visual loading efficiency of EPDNs models, it is necessary to simplify the $3 \mathrm{~d}$ models of different elements without affecting the display effect. This paper refers to the LOD classification idea of City GML, combined with the accuracy requirements and the complexity of the spatial geometry in different use case scenarios of EPDNs, and divides the EPDNs models into four levels of detail: LOD1-LOD4. At the LOD1, the electric power points are represented by spheres, whose centers are the geometrical centers of the spatial objects and tangent to their conterminous electric power lines; the electric power lines are represented by cylinders along their course; structures are represented by block models. At the LOD2, the geometric structures of various EPDNs elements are represented by coarser boundary surfaces. LOD3 adds materials and textures of elements to LOD2, and the EPDNs elements have more detailed structural surfaces. LOD4 adds internal structure of the elements to LOD3.

\subsection{CONSTRUCTION OF LOD MODELS FOR EPDNS}

This paper firstly studies the model criteria of various elements in EPDNs structural system, the geometric features that need to be paid attention to when modelling in $3 \mathrm{D}$, and the selection criteria of the geometric details of EPDNs models in the levels of detail mentioned in the previous section. Then this paper combines the topological relationship information between different elements of EPDNs to construct geometric models with different levels of detail. Since most of the EPDNs elements are irregular and complex buildings or structures, the automatic simplification algorithms of multi-detail hierarchical models that have been widely studied at present is not ideal and need to be studied for the kind of electric power models. In this paper, three-dimensional modelling software 3dsMax and CAD are selected. Taking the EPDNs data of the Yan Shan campus of Guilin University of Technology as an example, the multiple levels of detail models of different elements are set up by hand, and the EPDNs data models of multi-detail levels is constructed.

Because the EPDNs data are generally confidential, they are obtained based on field exploration, literatures and the network. For the electric power lines data, this paper simulates their layout and voltage grade according to the urban communities, and the diameters of them are set according to the national standards. The approximate outer diameters of the trunk cables are $63 \mathrm{~mm}$, and the approximate outer diameters of the branch cables are $29.8 \mathrm{~mm}$. They are obtained by performing a lofting operation on the spline in 3dsMax. For electric power point features and building structures, due to the complexity of their geometric structures, this paper models the real objects in accordance with 1:1, decomposes the complex point elements into simple geometry, and forms a complex model by geometric transformation and Boolean operation on the simple geometry in $3 \mathrm{dsMax}$. And then, this paper attaches a real texture map to the model. In the end, it is realized the EPDNs models that conform to both people's visual perception and multi-detail expression. The models of EPDNs data at the LOD1-LOD4 are shown in Figure 3. 


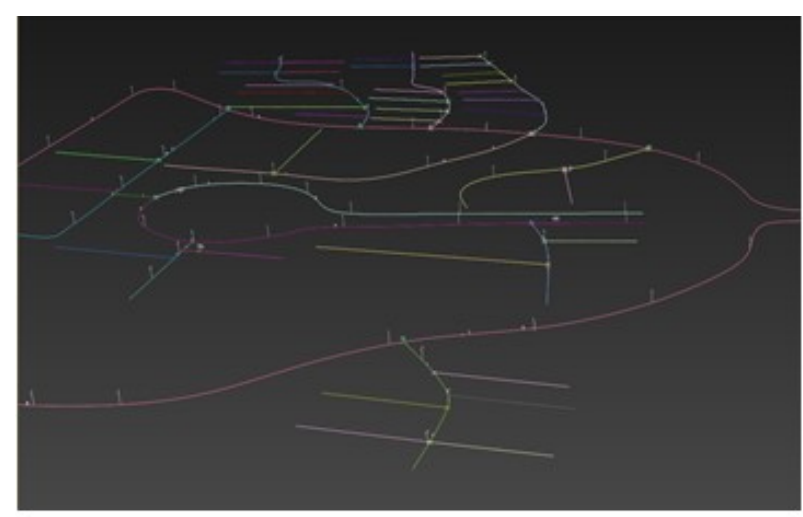

LOD1

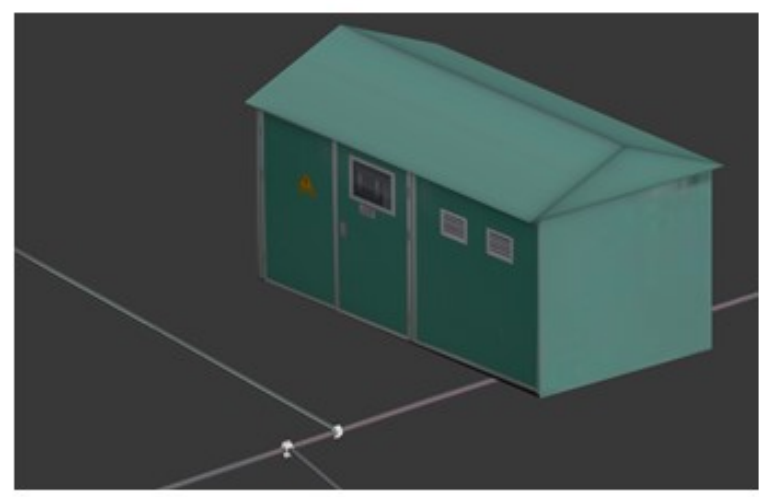

LOD3

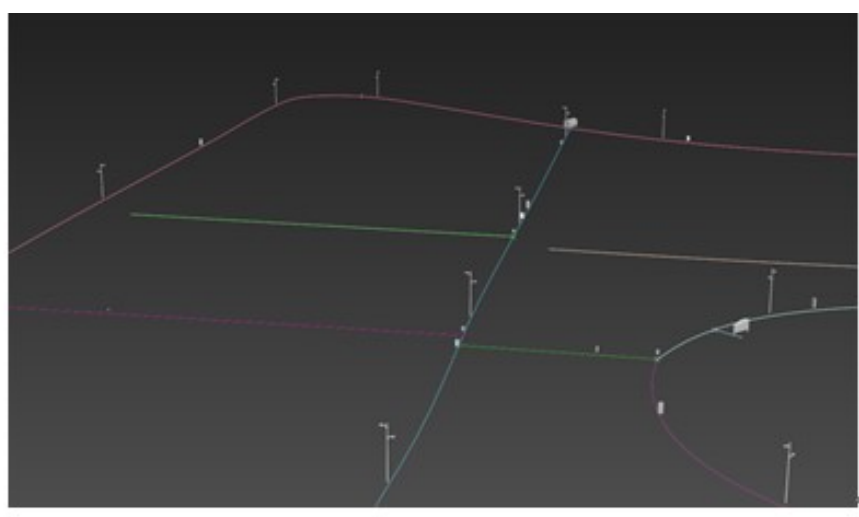

LOD2

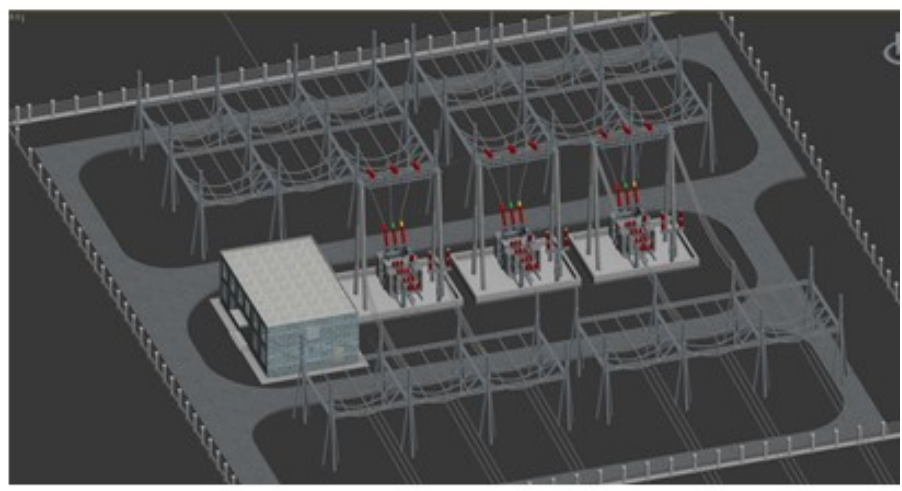

LOD4

Figure 3. Geometric expression of EPDNs models

\section{ANALYSIS OF EPDNS ATTRIBUTE DATA}

In the expression of multi-scale data, as the scale changes, the geometric shapes of spatial entity elements change, and at the same time, their attribute information also change significantly. For example, the transformer substation in life consists of primary equipment such as transformer and high-voltage circuit breaker. At a certain scale, the model is often simple and can only show that it is a transformer substation, but cannot refine its internal structure. As the scale increases, the transformer substation can be refined in spatial structure, and the attribute description also needs to divide the transformer substation into more detailed parts. In the process of transforming small-scale data into large-scale data, the spatial geometric representation of EPDNs elements is gradually refined, and the attribute information is gradually detailed. In small-scale scenarios, the electric power cable accessories may be discarded and not be displayed, and electric power lines are simplified to lines for presentation, some large and important ancillary facilities are also simplified as cuboids. At large scales, the electric power cable accessories can be displayed, electric power lines and ancillary facilities also exhibit a true geometric appearance, and their attribute information is more detailed. In the process of transformation from large-scale data to small-scale data, the EPDNs elements with different characteristics can generate objects with more advanced structure and functions. The former and the latter have inheritance in attributes, dependence in functions and generality in structure. But at the same time, the attributes which the high-level objects have, the lower-level objects do not necessarily have. For example, the transformer substation has the function of converting electric energy, but the high-voltage circuit breaker is not provided.
This paper analyzes and summarizes the attribute information of different types of EPDNs elements, and divides their attribute information into natural graphic attributes centered on elements and functional attribute centered on functions, and describes the attribute data contained in different EPDNs models in detail from the above two aspects:

(1) For the EPDNs graphic data at LOD1, electric power point elements only have general attribute information, the same is true for electric power lines and building structures. For example, the models of electric power points at LOD1 are represented by spheres, and it is impossible to distinguish whether they are cable wells or not. Therefore, the points data of this level only contains the basic attributes such as elevation, coordinates and buried depth, and does not contain the attributes of whether it is a well or not

(2) For the EPDNs graphic data at LOD2, the electric power point elements are expressed according to the information such as the purpose, angle of the symbol, spatial position and so on. The electric power line elements are described in terms of the type of power lines, core material, insulating material, buried mode, and long-distance lines. And the large building structures describe their attribute according to the type and service radius.

For the EPDNs graphic data at LOD3, the power-controlled device of the electric power points adds the information of type, that is, whether the device is a transformer or a control cabinet (box), and the powerdistributed device adds a role type, that is, whether the device is connected or functional. The above-ground element increases the attribute that whether it is a pole, 
and the underground element increases the attribute that whether it is a well. The direct buried cable in the electric power lines increases the attribute information of the cross-section size and the number of the cables. The non-direct buried cable increases the description of the total number of holes, the number of used holes, and the cross-section size. The overhead power line increases the number of hanging arms, circuits and the type. The primary equipment of the transformer substation, the size and composition of the switch board rooms and the voltage grade of the switch cabinets are described in detail in the building structures.

For the EPDNs graphic data at LOD4, the attribute information of point elements is added the type, phase number and insulating medium of the transformer, and the control cabinet material, the pole material, the joint type and wire core material of the electric power cable accessories, and the type, size and material of the cable well.

\section{ANALYSIS AND DISCUSSION OF MODELLING RESULTS}

According to the current technical regulations of urban underground pipeline detection and the relevant works of power industry, this paper makes a comprehensive and systematic summary of the constitution system of EPDNs. Based on the distribution of EPDNs elements in daily life and combined with EPDNs topological information, the multi-scale EPDNs data models are constructed. The EPDNs data models built in this paper contain reasonable graphical information and attribute information, covering the data requirements of users with different application backgrounds and different application modes in electric power operating maintenance, which can meet the visualization and spatial analysis of EPDNs data at different scales.

Because of the wide distribution range and complex spatial relationship of EPDNs, although we have conducted field investigations, the models still lack some details. It is necessary to investigate the professionals in electric power industry and improve the data models.

\section{CONCLUSIONS}

This paper takes EPDNs as the research object and studies their multi-scale spatial data models. The main research work and innovation of this thesis are as follows:

Based on the information of EPDNs, this thesis summarizes and sorts out the graphic data and attributive data of electric power elements in operation and maintenance fields, and then constructs a relatively complete constitution system of EPDNs.

Combining with the EPDNs architecture and visual expression mechanism, this research divides the EPDNs models into four levels based on the good visualization effect and less data redundancy, proposes the simplified principles of different level models and realizes the detailed description of the EPDNs graphic data and the attribute data at different levels.

Taking the EPDNs data of Yan Shan campus of Guilin University of Technology as an example, this study realizes the three-dimensional data modelling of different levels of detail, obtaining a good visualization effect.

\section{ACKNOWLEDGEMENTS}

The research work is supported by National Natural Science Foundation of China under Grant No.41861050.

\section{REFERENCES}

Cao, M.Z., 2017: Operation, maintenance and overhaul of power supply and distribution equipment. China Electric Power Press, Beijing.

Hu, Z.R., Zhu, G.R., Xu, Z.Y., 2006: Application Research of LOD Technology and Cartography Synthesis in Timely Display of Multi-scale Maps. Science of Surveying and Mapping (05), $78-79+47+6$

Jiang, S.S., 2017. Technical Specification for Urban Underground Pipeline Information Management System in Jiangsu Province[S]

Liu, H.M., 2005. Research on Technology of Level of Detail Model Simplification. Wuhan University of Technology, Wuhan, Hubei province, China.

Ning, B., 2012. Technical Specification for Pipeline Detection[S].

Qu, H.W., Li, Z.B., Li, H.J., 2018: Three-dimensional Simulation of Transmission Line Equipment Based on LOD Technology. Qinghai Electric Power 37(02), 32-37.

Su, Z., 2007. Data Format Standard of Urban Comprehensive Underground Pipeline in Suzhou[S].

Shen, Z., 2015. Standard for Data Construction of Underground Pipeline in Shenzhen[S].

Wu, D.H., Mao, X.C., Liu, Y., 2005: A Survey of 3D Spatial Data Models. Engineering of Surveying and Mapping 14(3), 70-73.

Wu, J., Ma, X.H., 2006: A Novel Method for Generating Multiple LOD Models of 3D Mesh. Journal of Nantong University (Natural Science) (03), 33-36.

Zhu, G.M., Ma, Z.T., Sun, L.X., 2007: Research on Fast Construction Method of 3DModels in 3DUGIS. Geography and Geo-Information Science 23(4), 29-32+40.

Zhao, J.Q., 2013: Automatic Simplification Approach for the LODs of Complex 3D Building Models. Acta Geodaetica et Cartographica Sinica 42(01), 156.

Zhu, Q., Gong, J., Du, Z.Q., Zhang, Y.T., 2005: LODs Description of 3D City Model. Editorial Board of Geomatics and Information Science of Wuhan University (11), 28-32.

Zhou, Y., Zhu, Q., Huang, Y., 2006: LOD of Complex Buildings for 3DCM. Science of Surveying and Mapping (05), 74-77+6. 